\title{
Delay-aware tree construction and scheduling for data aggregation in duty-cycled wireless sensor networks
}

\author{
Duc Tai Le1, Taewoo Lee ${ }^{2}$ and Hyunseung Choo ${ }^{1,2^{*}}$
}

\begin{abstract}
Data aggregation is one of the most essential operations in wireless sensor networks (WSNs), in which data from all sensor nodes is collected at a sink node. A lot of studies have been conducted to assure collision-free data delivery to the sink node, with the goal of minimizing aggregation delay. The minimum delay data aggregation problem gets more complex when recent WSNs have adopted the duty cycle scheme to conserve energy and to extend the network lifetimes. The reason is that the duty cycle yields a notable increase of communication delay, beside a reduction of energy consumption, due to the periodic sleeping periods of sensor nodes. In this paper, we propose a novel data aggregation scheme that minimizes the data aggregation delay in duty-cycled WSNs. The proposed scheme takes the sleeping delay between sensor nodes into account to construct a connected dominating set (CDS) tree in the first phase. The CDS tree is used as a virtual backbone for efficient data aggregation scheduling in the second phase. The scheduling assigns the fastest available transmission time for every sensor node to deliver all data collision-free to the sink. The simulation results show that our proposed scheme reduces data aggregation delay by up to $72 \%$ compared to previous work. Thanks to data aggregation delay reduction, every sensor node has to work shorter and the network lifetime is prolonged.
\end{abstract}

Keywords: Data aggregation scheduling, Collision-free, Duty cycle, Wireless sensor networks

\section{Introduction}

Wireless sensor networks (WSNs) are generally composed of a large number of sensor nodes cooperating with each other to conduct various services, such as monitoring a disaster area in environmental services, detecting event on a barrier in military surveillance services, gathering a patient information in health care services, and so on [1]. Each sensor node detects events and periodically sends the sensory data to a sink node (base station). Data aggregation is a process of collecting all data to the sink node, where data packets can be aggregated at intermediate nodes along the multi-hop paths toward the sink to reduce the number of packets and thus conserve the energy for transmissions [2]. Such aggregation operations can be realized by eliminating duplicate packets and combining

\footnotetext{
${ }^{*}$ Correspondence: choo@skku.edu

${ }^{1}$ Convergence Research Institute, Sungkyunkwan University, Jangangu,

Seobu-ro 2066, 440-746 Suwon, Republic of Korea

${ }^{2}$ College of Software, Sungkyunkwan University, Jangangu, Seobu-ro 2066,

440-746 Suwon, Republic of Korea
}

multiple packets into a single packet [3]. Like other operations in a wireless medium, data aggregation suffers from collision when a node simultaneously hears more than one packets from its neighbors. The collision results in increasing not only the number of transmissions but also the delay. The additional delay may become serious in real-time application when a node fails to forward data by an appointed time. Thus, the collision problem should be taken into account to ensure the reliability of data aggregation.

Several objectives of data aggregation have been pursued according to the diverse requirements of applications, such as energy efficiency $[4,5]$, maximum network lifetime [6,7], maximum quality of aggregation [8,9], and minimum delay [10-12]. Among these objectives, minimizing data aggregation delay has risen as one of the most important problems in WSNs and has been widely studied. The problem gets more complex when recent WSNs have adopted the duty-cycle scheme to conserve energy and to extend network lifetime [13, 14]. In 
a duty-cycled WSN, every sensor node periodically alternates between active and sleeping states. In addition to a reduction of energy consumption, the periodic sleeping of sensor nodes yields a notable increase in communication delay compared with that of always-active networks [15-22]. This is because a sensor node has to wait until its receiver wakes up before transmitting a packet. Existing data aggregation schemes for always-active WSNs cannot be directly applied to duty-cycled networks.

Yu et al. [23] claim the NP-hardness of the minimum time aggregation scheduling problem in duty-cycled WSNs, later on referred to as dc-MTAS problem, and have presented an aggregation scheduling algorithm. The algorithm, named SA, deploys a minimal covering schedule on a layered network structure to minimize aggregation delay. It utilizes connected dominating set (CDS) information in the aggregation process to reduce the possibility of collision, thus reducing the number of transmissions. SA algorithm produces a collision-free data aggregation schedule for duty-cycled WSNs in a polynomial running time.

This paper proposes a latency efficient data aggregation scheduling scheme for the dc-MTAS problem. The proposed scheme consists of two phases: CDS-based tree construction and first-fit scheduling. The tree construction phase first forms a CDS for a duty-cycled WSN. It then takes the sleeping delay between a pair of neighboring nodes into account to build a delay-aware data aggregation tree based on the CDS. The scheduling phase guarantees collision-free data aggregation on the constructed tree. It seeks the fastest available transmitting time slot for each node in the network to minimize aggregation delay. The contributions of this paper can be summarized as follows:

- We present a novel scheduling scheme for minimizing data aggregation delay in duty-cycled WSNs. The scheme does not allow any collision in a schedule to conserve energy for transmission.

- We analyze and prove the correctness and time complexity of the algorithms in the proposed scheme.

- We conduct in-depth simulation in various scenarios to evaluate the effect of our proposed algorithms on aggregation delay. The simulation results show that the proposed scheme improves up to $63 \%$ with different node density, $60 \%$ with different duty cycles, and $72 \%$ with different transmission ranges, compared to existing schemes.

The rest of this paper is organized as follows. We discuss related works in Section 2. Section 3 includes the problem statement and network model under consideration. The proposed scheme is presented in Section 4. We analyze the correctness and time complexity of the scheme, and then evaluate its performance in Section 5. Finally, we conclude the paper and discuss our future work in Section 6. Finally, we conclude the paper and discuss our future work in Section 7.

\section{Related work}

The problem of minimum time aggregation scheduling, which is essential to enable real-time ability of WSNs, has been extensively studied in literature [10-12]. Chen et al. [10] have claimed the NP-hardness of the problem, and proposed the shortest data aggregation (SDA) algorithm. The algorithm consists of two phases: tree construction and data aggregation scheduling. It schedules a data aggregation process in the second phase, based on the shortest path tree (SPT) constructed in the first phase. However, the schedule results in an inefficient data aggregation delay because the SPT construction leads to high-degree nodes in the tree. SDA algorithm has the approximation ratio of $(\Delta-1) R$, where $\Delta$ and $R$ are the maximum degree and the graph-theoretic radius of a WSN, respectively.

Huang et al. [11] presented a first-fit scheduling for data aggregation based on a CDS tree. To construct the tree, firstly, all nodes in a WSN are divided into layers based on a breadth first search (BFS) tree. Secondly, some nodes are selected as dominators based on a maximal independent set (MIS). Such dominators are then connected by connectors to form the CDS tree. Finally, the remaining nodes become dominatees of the network. However, the first-fit scheduling only guarantees that every transmission from a node to its parent in the tree is collision-free. It can result in collision possibly occurring at a neighbor of the node in the network. The approximation algorithm has the delay bounded by $23 R+\Delta-18$. The upper bound has been improved to $16 R+\Delta-14$ [12].

The above algorithms assume an always-active network and are not suitable for a duty-cycled network because of its intermittently connected characteristic [13, 14]. Due to the periodic sleeping of every node in duty-cycled WSNs, minimizing delay is one of the most important issues in such networks [15-22]. In order to reduce the latency of a data aggregation in duty-cycled WSNs, several approaches have been proposed. For networks with adjustable duty-cycles, DMAC [24] adjusts the duty cycles adaptively according to the traffic load in a WSN to provides significant energy savings and latency reduction. Gu et al. [25] introduced Spatiotemporal Delay Control, which increases duty cycle at individual nodes and optimizes the position of sink nodes, to reduce communication delay.

For the dc-MTAS problem, scheduling algorithm (SA) [23] is one of the earliest schemes. The scheme consists of two phases. In the first phase, the layered structure construction (LSC) algorithm forms a CDS of a duty-cycled 
WSN based on an MIS. Nodes that are not included in the CDS become dominatees of the network. The algorithm then builds a layered virtual backbone for data aggregation based on the CDS. However, LSC algorithm does not consider the delay caused by the periodic sleeping of nodes in the network. Thus, it results in a backbone with the longest path delay in the worst case. In the second phase, the working period scheduling (WPS) algorithm first collects data from all dominatees to dominators in CDS. The data is then transmitted layer-by-layer from the dominators to a sink through the backbone. To guarantee that a node in layer $i$ transmits data after all nodes in layers deeper than $i$ finish transmitting data, the algorithm delays the working period of all nodes in layer $i$ by the largest working period of nodes in the deeper layers. This leads to an extra delay for every transmission in layer $i$, which makes the overall aggregation delay of SA scheme high.

On minimizing data aggregation delay, we have discovered that constructing an aggregation tree with a preference of links with small sleeping delays is beneficial to reduce aggregation delay. The intuition behinds this is that the smaller sleeping delay a link has, the shorter time period a transmission is delayed on the link. Such an aggregation tree construction has been sketched in [26] by an algorithm using both topology and duty-cycling information of a duty-cycled WSN. Based on the constructed tree, data is collected to a sink node by the first-fit aggregation scheduling. We have presented preliminary simulation results to show the advantage of the delay-aware tree construction in reducing aggregation delay.

In this paper, we provide a comprehensive description of the delay-aware aggregation tree construction with refined pseudo code and a complete example. Additionally, we improve the aggregation scheduling by separating it into two steps: dominatee collection and backbone aggregation. Such separation makes the scheduling more practical and natural to ensure a collision-free aggregation. The extended simulation results demonstrate a significant improvement of the proposed scheme on aggregation delay. Last but not least, the correctness of the proposed scheme and its complexity are analyzed in the paper.

\section{Preliminary}

\subsection{Network model}

We consider a static WSN including uniformly deployed sensor nodes and one randomized sink node. Each node is equipped with an omni-directional antenna, and all nodes have the same transmission range. Two nodes become neighbors to each other and can communicate if they are within their transmission ranges. The network topology can be modeled as an undirected graph, in which each vertex and each edge correspond to a node and a communication link between two neighbor nodes in the network, respectively. Such a graph is called a communication graph and assumed to be connected.

In a duty-cycled WSN, each node has two possible states: active state and sleep state [14]. Each state is determined by turning on or off the RF transceiver of a node. In this paper, we assume that each node determines the active and sleep states independently, when it is deployed [27]. The entire lifetime of each node is divided into multiple working periods of the same length, each of which has $\tau$ time slots. All sensor nodes are time synchronized at the slot level using local time synchronization techniques, such as Flooding Time Synchronization Protocol [28] and Tiny-Sync [29]. Each node $u$ randomly selects one time slot of $a(u) \in[0, \tau-1]$ as its active slot and sleeps in the remaining $\tau-1$ time slots in a working period. The duty cycle is defined as $1 / \tau$. The duration of each time slot is long enough for a node to send or receive one data packet. A node can receive data only in its active time slot, but can transmit a data packet at any time slot.

We assume that every node in WSNs uses the halfduplex mode, i.e., it can either send or receive one data packet in one time slot. In other words, the node cannot send and receive at the same time. We further assume the interference range and the transmission range of each node are the same [30]. The interference causing a packet collision is classified into two types: primary and secondary interferences. Primary interference occurs when a node has more than one communication task in a single time slot. Typical examples are sending and receiving at the same time, or receiving from two different nodes. Secondary interference occurs when a node that is receiving a data packet overhears another transmission intended for other nodes. Our proposed scheme prevents collisions caused by both primary and secondary interferences in duty-cycled WSNs.

Figure 1 shows an example of the two types of interference that can occur in data aggregation scheduling, based on our assumption. A letter and a number in each node represent its node ID and active slot, respectively. Each working period is composed of four time slots, i.e., duty cycle $25 \%$. A black slot represents active state, and a white slot represents sleep state. Data from all nodes is collected to the sink node $a$. If nodes $d$ and $e$ send data to node $b$ in the same working period, a collision, caused by primary interference, happens at node $b$. A secondary interference, resulting in a collision at node $c$, occurs if nodes $e$ and $f$ send data to nodes $b$ and $c$ in the same working period, respectively.

\subsection{Problem formulation}

We consider an aggregation process gathering data from all nodes in a duty-cycled WSN to a sink node. Each sensor node in the network sends a single packet containing 


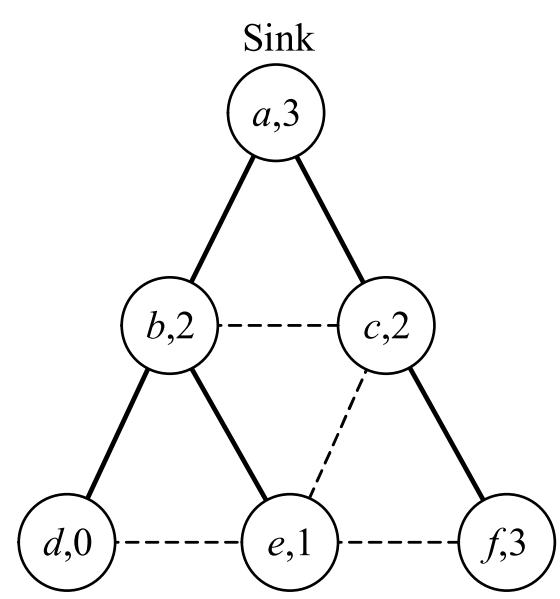

a

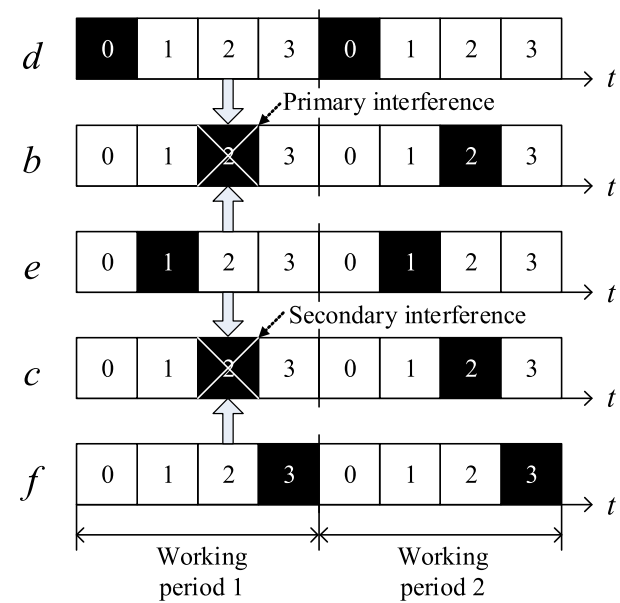

b

\begin{tabular}{|llll|}
\hline$i, j$ Node $i$ with active slot $j$ & Sleeping slot & Active slot \\
$\searrow$ Packet collision & --- Network link & Data aggregation tree edge
\end{tabular}

Fig. 1 An example of collision in data aggregation scheduling in duty-cycled WSNs. a Network topology. b Duty-cycled schedule

its own data and data received from neighbors toward the sink node through a multi-hop path. A complete data aggregation function, such as COUNT, MIN, MAX, SUM, and AVERAGE, is deployed to ensure that every packet has the same size [2]. An aggregation schedule assigns a working period and a receiving node to every node, except the sink, for transmitting a data packet. The scheduled transmission must be collision-free while the maximum assigned working period is minimized.

In this paper, let $G=(V, E)$ denote the communication graph of a duty-cycled WSN, where $V$ is the set of vertices, and $E$ is the set of edges. We denote a sink node and the set of scheduled senders sending data in working period $m$ by $s \in V$ and $S_{m}$, respectively. An aggregation schedule is defined as $S=\bigcup_{m=1}^{D} S_{m}$, where $D$ is the aggregation delay to the sink. The aggregation delay is determined by the largest value of scheduled working period $m$, among all nodes in the WSN. The dc-MTAS problem is formally defined as:

Finding a data aggregation schedule $S$ which minimizes $D$ s.t.

(i) $S=\bigcup_{m=1}^{D} S_{m}=V \backslash\{s\}$.

(ii) $S_{m} \cap S_{n}=\emptyset, \forall m \neq n$.

(iii) Any pair of transmissions of senders in $S_{m}(1 \leq m \leq D)$ are not conflicting.

(iv) All data are aggregated to the sink $s$ in $D$ working periods.

The dc-MTAS problem is an NP-hard problem [23].

\section{Delay-aware data aggregation scheduling}

We present details of our proposed data aggregation scheduling in this section. The scheme has two phases: the layered network structure construction phase and collision-free scheduling phase. The first phase, delayaware tree construction (DTC), builds a CDS tree taking the delay between nodes in a duty-cycled WSN into account and prepares a virtual backbone for efficient data aggregation in the network. The second phase, first-fit aggregation scheduling (FAS), conducts a fast collisionfree data aggregation schedule based on the constructed backbone. Table 1 shows the notations used in this paper.

\subsection{Delay-aware tree construction}

As shown in Algorithm 1, DTC algorithm is composed of three steps. In step 1 , it constructs a BFS tree rooted at sink node $s \in V$ of a network $G=(V, E)$ (line 1$)$. All nodes in the network are divided into layers $L_{0}, L_{1}, \ldots$, and $L_{l_{\max }}$ according to the hop distance from $s$ (line 2), where $l_{\max }$ is the largest layer in the BFS tree. Layer $L_{0}$ contains only the sink node. The sink is initially selected as a dominator and added to a dominating set $D S$ (line 3 ). The algorithm executes multiple iterations of steps 2 and 3 from layer 1 to layer $l_{\max }$ to construct a CDS tree rooted at $s$. To facilitate the delay-aware tree construction, DTC algorithm determines the sleep delay between every pair of nodes $(u, v)$ as follows:

$$
d(u, v)= \begin{cases}a(v)-a(u), & \text { if } a(v)>a(u) \\ a(v)-a(u)+\tau, & \text { otherwise }\end{cases}
$$


Table 1 Notation

\begin{tabular}{|c|c|}
\hline$N(u)$ & Set of neighbor nodes of node $u$ \\
\hline$a(u)$ & Active slot of node $u$ \\
\hline$\tau$ & The number of time slots in a working period \\
\hline$L_{i}$ & Set of nodes in layer $i$ \\
\hline$T_{C D S}$ & Connected dominating set tree \\
\hline$C D$ & Set of candidate dominators \\
\hline DS & Set of dominators \\
\hline$P(u, v)$ & The shortest path in terms of delay from nodes $u$ to $v$ \\
\hline$d(u, v)$ & Delay from nodes $u$ to $v$ \\
\hline$d 2 d(u)$ & $\begin{array}{l}\text { The smallest delay from node } u \text { to upper dominator within } \\
\text { 2-hop }\end{array}$ \\
\hline$p(u)$ & Parent of node $u$ in $T_{C D S}$ \\
\hline$O x(u)$ & Set of overhearing working periods of node $u$ \\
\hline$\overline{T x}(u)$ & $\begin{array}{l}\text { Set of forbidden transmission working periods of node } u \text { to } \\
\text { avoid collision }\end{array}$ \\
\hline$r x(u)$ & The last receiving working period of node $u$ \\
\hline $\operatorname{tx}(u)$ & Schedule for transmission of node $u$ \\
\hline
\end{tabular}

The $d(u, v)$ shows the delay for which node $u$ has to wait to send data to node $v$, at the active time slot of node $v$. The sleep delay of a path is the accumulated sleep delays of all edges in the path.

Let $L_{i}$ be the set of nodes in layer $i(1 \leq i \leq l)$. In step 2 , nodes in $L_{i}$, which are not adjacent with any dominator, become candidate dominators and are added to set $C D$ (line 5). The shortest paths in terms of delay from a candidate dominator to all dominators within two-hop distance are established to identify the nearest dominator of the candidate (line 7). Such a dominator always exists in the upper layer $j(0 \leq j<i)$ because of the network connectivity. The shortest path from a candidate dominator to its nearest dominator, and the sleep delay of the path are recorded for further use (lines 8-9). Let $P(u, v)$ denote the shortest path in terms of delay between candidate dominator $u$ and its nearest dominator $v$. The sleep delay of $P(u, v)$ is $d 2 d(u)=d(u, w)+d(w, v)$, where $w$ is the intermediate node of the path.

DTC algorithm selects dominators among the candidates and then builds a CDS tree $T_{C D S}$ to connect all the dominators in step 3 . Initially, the tree contains only sink node $s$. In each layer, node $u \in C D$ is selected to be a dominator if it has the smallest value of $d 2 d(u)$ among all nodes in the candidate dominator set. The selected dominator and all of its neighbors are removed from the set (lines 11-12). Node $u$ and the intermediate node $w$ on the path $P(u, v)$ are added to $T_{C D S}$ (lines 13-14). Node $w$ is referred to as a connector and becomes the parent node $p(u)$ of node $u$ in the tree. It is worth noting that the nearest dominator $v$ of node $u$ is already in $T_{C D S}$ and becomes

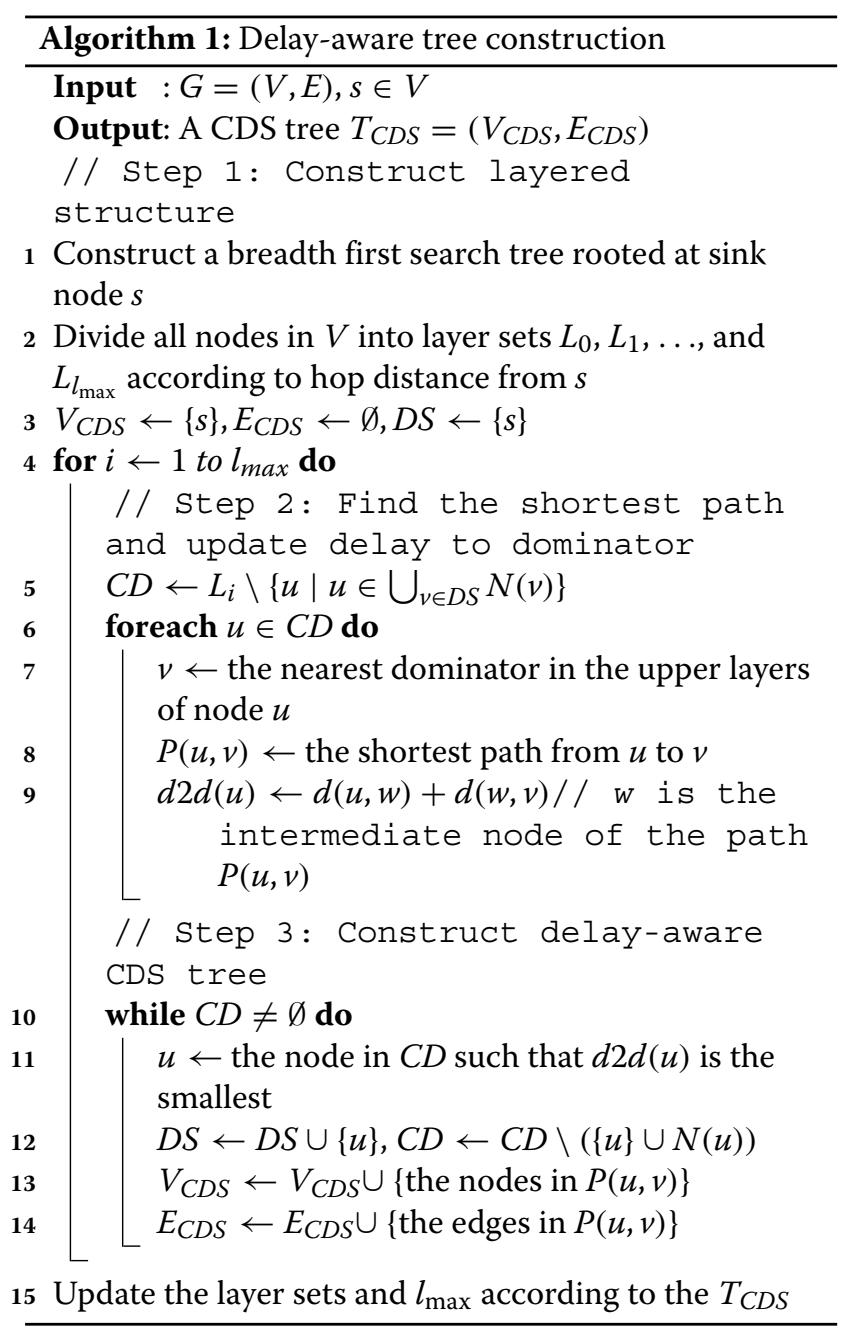

$p(w)$. DTC algorithm iterates step 2 and step 3, layer-bylayer, until the layer $l_{\max }$. It then updates the layer sets and $l_{\max }$ such that every node is in the adjacent lower layer of its parent in $T_{C D S}$ (line 15). We refer to nodes that are not in the CDS tree as dominatees.

Figure 2 shows an example of DTC algorithm on a network of 13 nodes with a duty cycle of $25 \%(\tau=4)$. Nodes in the network are divided into layers as shown in Fig. 2a. Initially, sink node $a$ is selected as a dominator and added to the tree. All nodes in layer 1 become dominatees as they are adjacent to $a$. All nodes in $L_{2}=\{e, f, g, h\}$ become candidate dominators. The delays of all paths from a candidate dominator to node $a$, the two-hop upper dominator, are shown in Fig. 2b. The algorithm determines the shortest path in terms of delay and calculates the delay to the nearest upper dominator for each node in $L_{2}$, as in Fig. 2c. Node $f$ is first selected as a dominator because it has the smallest delay to the upper dominator of $d 2 d(f)=3$. Nodes $e$ and $g$ in $L_{2}$ become dominatees as they are adjacent to $f$. In the same manner, node $h$ becomes 

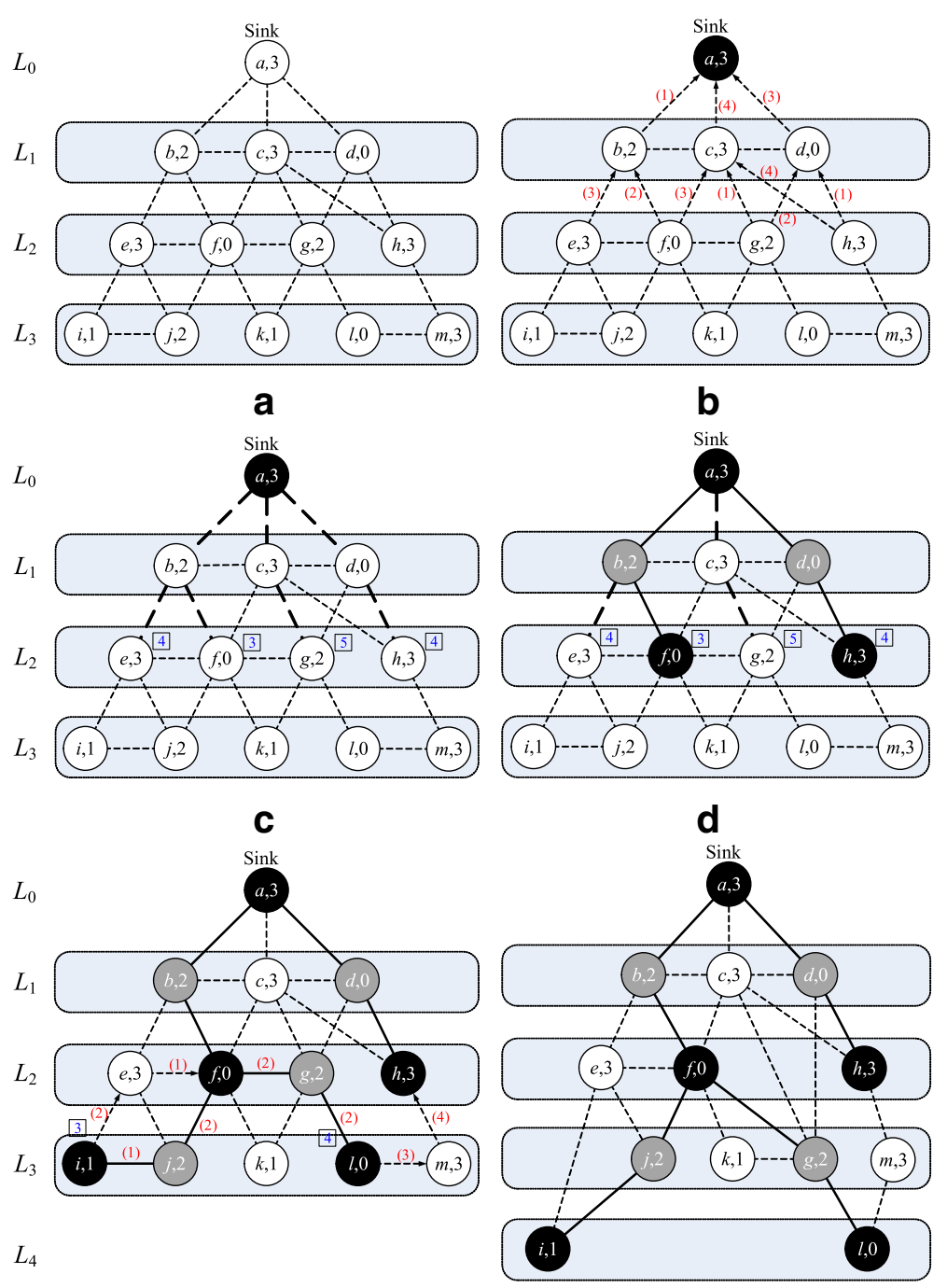

$L_{4}$

f

\begin{tabular}{|c|c|}
\hline Node $i$ with active slot $j$ & Dominator \\
\hline $\begin{array}{l}(i, j) \stackrel{(n) \rightarrow}{\rightarrow}\left(i^{\prime}, i^{\prime}\right) \text { Delay from } i \text { to } i^{\prime}: n \\
-- \text { The shortest 2-hop path link }\end{array}$ & $\begin{array}{l}n \text { Delay to dominator }----- \text { Communication link } \\
-T_{C D S} \text { edge } \square \text { Layer information }\end{array}$ \\
\hline
\end{tabular}

Fig. 2 An example of delay-aware tree construction. a Step 1: constructing layered structure. b Step 2 in $L_{2}$ : calculating delay from candidate dominator to upper dominators. c Step 2 in $L_{2}$ : finding the shortest path in terms of delay. d Step 3 in $L_{2}$ : adding dominators and connectors to the CDS tree. e Step 2 and step 3 in $L_{3}$. $\mathbf{f}$ Updating layer sets according to $T_{C D S}$

a dominator. In Fig. 2d, the dominators $f$ and $h$ are connected to the CDS tree by the two connectors of $b$ and $d$, respectively. Figure 2e illustrates the process of DTC algorithm for nodes in layer 3. Finally, in Fig. 2f, the layer sets are updated based on the CDS tree. The layers of nodes $g, i$, and $l$ are increased to be bigger than those of their corresponding parents.

\subsection{First-fit aggregation scheduling}

Referring to the CDS tree $T_{C D S}$ of a WSN as an aggregation backbone, the FAS scheme constructs a delay-efficient schedule, gathering data from all nodes in the network to the sink. The scheduling scheme consists of two steps. It first collects data from all dominatees to nodes in the backbone and then collision-free aggregates the data from the backbone nodes to the sink in a bottom-up manner. It is worth noting that the backbone nodes include all dominators and connectors selected in DTC algorithm.

The pseudo code of dominatee scheduling algorithm for the dominatee data collection is shown in Algorithm 2. According to $T_{C D S}$, all nodes in a network are separated 
into two disjoint sets $R$ and $S$, the set of nodes in the backbone and the set of dominatees, respectively (line 1). Each dominatee in $S$ is adjacent to at least one backbone node in $R$, i.e., its dominator. Starting from working period 1 , the algorithm assigns a working period for each sending node in $S$ so that its data packet can be delivered collision-free to a receiving node in $R$. The assigned working periods should be as small as possible to enable a minimum delay aggregation.

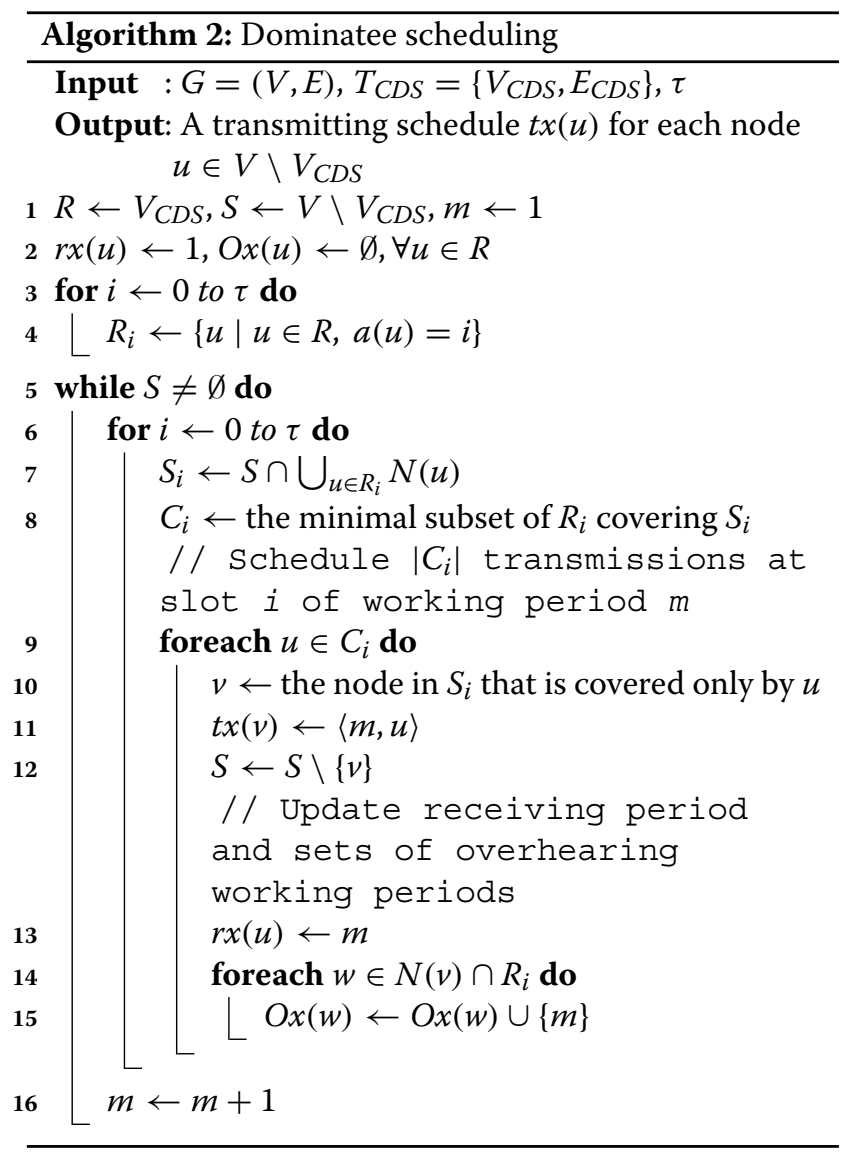

To ensure collision-free data collection, dominatee scheduling groups the receiving nodes into different subsets $R_{i}$, where $0 \leq i<\tau$, according to their active slots (lines 3-4). Let $S_{i}$ denote the subset of sending nodes adjacent to nodes in $R_{i}$ (line 7). Algorithm 2 searches for a minimal subset $C_{i} \subset R_{i}$ that can cover all nodes in $S_{i}$ (line 8). According to the property of the minimal cover set, each node $u \in C_{i}$ must have at least one proprietary neighbor $v \in S_{i}$, which is only adjacent to $u$ (line 10). If a receiving node has more than one proprietary neighbor, the algorithm randomly selects one of the neighbors as a sending node. We denote the transmitting schedule of node $v \in S_{i}$ by $t x(v)=\langle m, u\rangle$ (line 11), meaning that node $v$ is scheduled to send a data packet to node $u$ at time slot $a(u)=i$ of the $m$-th working period. Obviously, there are $\left|C_{i}\right|$ such pairs of neighboring nodes in each active slot $i$. The transmissions between the pairs of nodes can be scheduled simultaneously in the same working period $m$ without any collision.

After dominatee $v$ is scheduled to transmit its data packet, it is removed from the sending node set $S$ (lines 11-12). Although only the corresponding receiving node $u \in C_{i}$ is ensured to receive the packet in working period $m$ (line 13), all nodes in $N(v) \cap R_{i}$ can overhear the transmission because they are active at the same time slot $i$. Algorithm 2 records the overheard working period for the neighbor nodes to prevent later transmissions from interfering (lines 14-15). The remaining dominatees will be scheduled iteratively at the next active slots, and then the next working periods, until all dominatees are scheduled (line 16). It is worth noting that transmissions at different active slots will not interfere with each other.

Figure 3 illustrates the dominatee scheduling algorithm on the same network as the example of DTC algorithm in Section 4.1. Recall that a working period has four time slots, i.e., $\tau=4$, in this example. According to the constructed $T_{C D S}$ shown in Fig. 3a, all nodes are separated into a sending set $S=\{c, e, k, m\}$ and a receiving set $R=$ $\{a, b, d, f, h, j, g, i, l\}$, as in Fig. 3b. The Algorithm 2 schedules transmissions from nodes in $S$ to nodes in $R$ at each active slot, iteratively. At active slot 0 , only nodes in $R_{0}=$ $\{d, f, i\}$ can receive data packets from their neighbors in $S_{0}=\{c, e, k, m\}$. A minimal cover set $C_{0}=\{f, l\} \subset R_{0}$ is constructed to cover all nodes in $S_{0}$. There are $\left|C_{0}\right|=2$ transmissions to receiving nodes $f$ and $l$ from their corresponding proprietary neighbors $c$ and $m$ scheduled in the same working period, as in Fig. 3c. In the same manner, the algorithm schedules transmissions from the remaining sending nodes $e$ and $k$ to the receiving nodes $i$ and $g$ at active slots 1 and 2, as shown in Fig. 3d, c, respectively. The dominatee data collection process completes, since all dominatees have been scheduled to transmit their data to nodes in the $T_{C D S}$, as in Fig. $3 f$.

The second step of FAS scheme starts after collecting all data from dominatees. The pseudo code of backbone scheduling algorithm for aggregating data from dominators and connectors is shown in Algorithm 3. The algorithm determines the first available working period for every node to transmit a data packet to its parent in the constructed $T_{C D S}$ layer-by-layer. Because a node can receive only in active state, if node $u$ is active before node $p(u)$ in a working period, it can then transmit a packet to $p(u)$ in the same working period of its last receiving one, i.e., $r x(u)$. Otherwise, it has to wait until $p(u)$ is active in the next working period (lines 3-4). It is worth noting that a node is ready to transmit after receiving all data from not only its children in the second step, but also its dominatess 


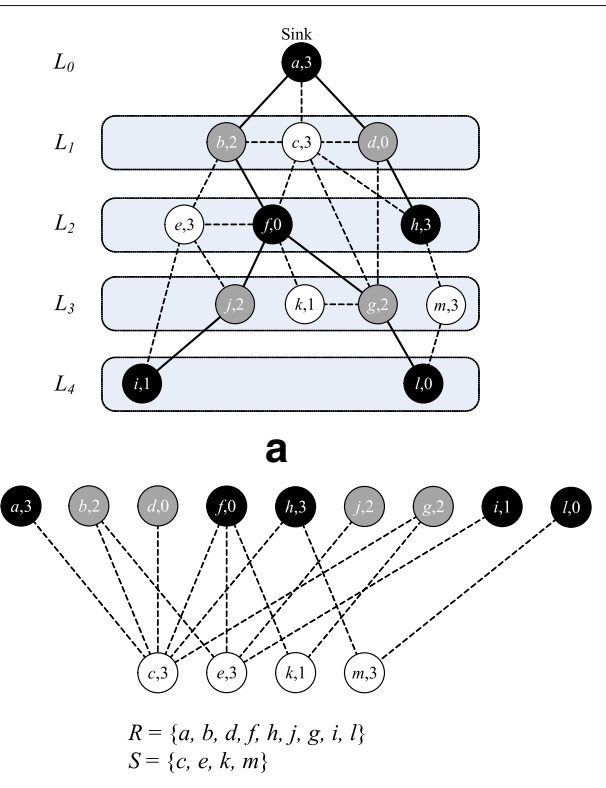

b

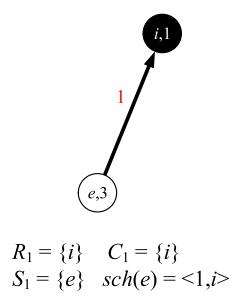

d

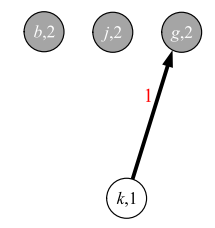

$R_{2}=\{b, j, g\} \quad C_{2}=\{g\}$ $S_{2}=\{k\} \quad \operatorname{sch}(k)=<1, g>$

e

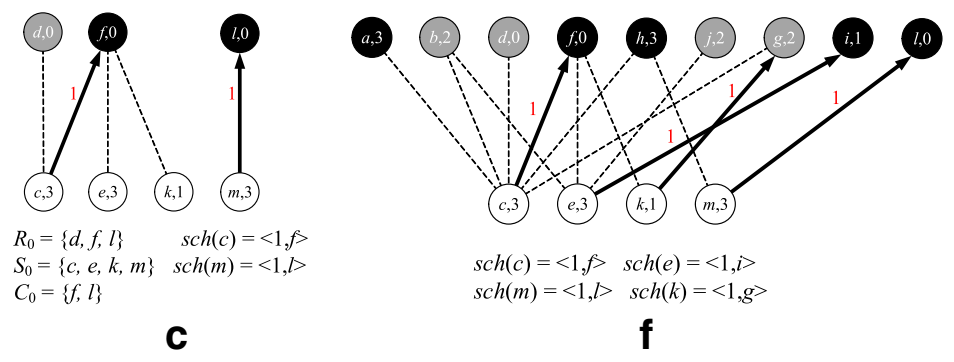

$(i, i)$ Node $i$ with active slot $j \bigcirc$ Dominatee $\bigcirc$ Dominator $\bigcirc$ Connector $\square$ Layer information
$(i, j) \rightarrow\left(i^{\prime}, i^{\prime}\right)$ Transmission from $i$ to $i^{\prime}$ in $n^{\text {th }}$ working period -----.- Communication link $-T_{C D S}$ edge

Fig. 3 An example of dominatee scheduling. a Layered network structure. b Separating nodes into receiving and sending sets. c Scheduling at active slot 0, working period 1. d Scheduling at active slot 1, working period 1. e Scheduling at active slot 2, working period 1. f Transmissions in the same working period

in the first step. A leaf node, which did not receive any data from dominatees, is ready at working period 1 .

Note that all neighbors of node $u$ active at the same time slot with node $p(u)$ can overhear a transmission from $u$ to the parent node. The transmission of $u$ may result in collisions at the neighbor nodes by interfering with other scheduled transmissions. To ensure collisionfree data aggregation, the backbone scheduling algorithm collects all working periods in which such neighbors in $T_{C D S}$ of node $u$ overhear other scheduled transmissions. It refers to such working periods as forbidden working period of $u$ and stores them in $\overline{T x(u)}$ (lines 5-6). The algorithm prevents node $u$ from transmitting a packet in the working periods and searches for the minimum transmitting working period for $u$ (lines 7-8). The last receiving working period of $p(u)$ and overhearing working periods of all neighbors active at $a(p(u))$ in $T_{C D S}$ of node $u$ are updated for further collision avoidance. Algorithm 3 assigns a first-fit working period for each node in the tree in a bottom-up manner so that data from all nodes is aggregated at the sink node.

Figure 4 shows the process of the backbone scheduling algorithm on the same network as the example of DTC algorithm in Section 4.1. According to the dominatee data collection schedule in Fig. 3f, transmissions of the dominatees are redrawn in Fig. 4a. The overhearing working periods caused by the transmissions are updated for backbone nodes, as shown in Fig. 4b. The Algorithm 3 starts from nodes in $L_{4}=\{i, l\}$, as shown in Fig. 4c. Since the overhearing working period sets of all neighbors of node $i$ are empty, the forbidden set $\overline{T x(i)}=\emptyset$. As node $i$ is active before its parent $j$ in a working period, $i$ can transmit a packet to $j$ in the same working period 1 , when receiving from dominatee $e$. On 

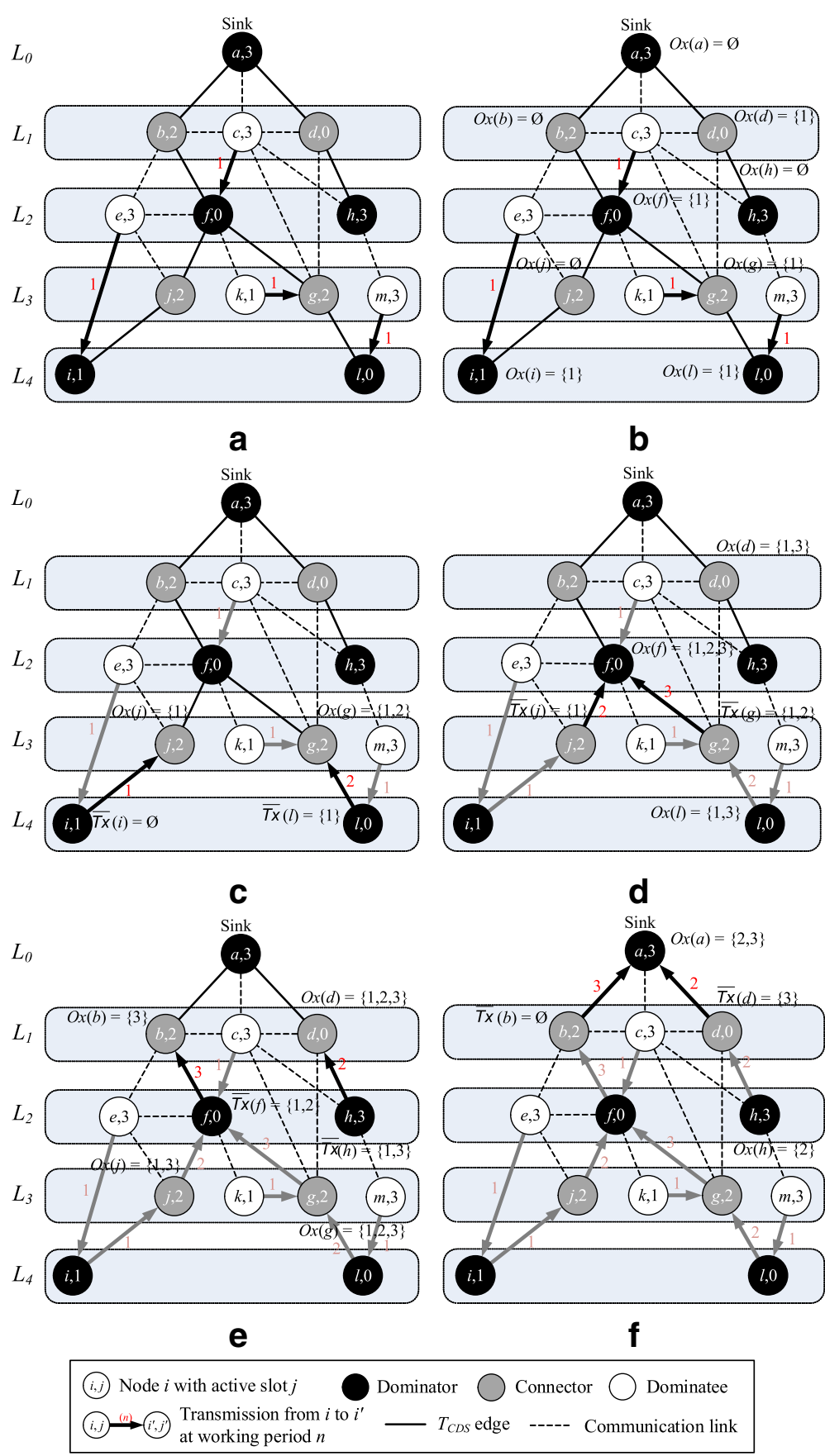

Fig. 4 An example of delay-aware tree construction. a Scheduling dominatees to nodes in $T_{\text {CDS }}$. b Overhearing periods after dominatees scheduling c Scheduling for nodes in $L_{4}$ of $T_{C D S}$. d Scheduling for nodes in $L_{3}$ of $T_{C D S}$. e Scheduling for nodes in $L_{2}$ of $T_{C D S}$. f Scheduling for nodes in $L_{1}$ of $T_{C D S}$

the other hand, node $l$ delays its transmission to working period 2 because its parent node $g$ is busy with a scheduled transmission from dominatee $k$ in working period 1 . The schedules of transmissions from nodes in $L_{3}, L_{2}$, and $L_{1}$ are shown in Fig. $4 \mathrm{~d}-\mathrm{f}$, respectively. The data aggregation schedule in this example completes at working period 3.

\section{Results and discussion}

\subsection{Theoretical analysis}

\subsubsection{Correctness}

Our proposed scheme provides a collision-free data aggregation scheduling in duty-cycled WSNs. The scheme reduces aggregation delay by deploying a first-fit schedule on a constructed CDS tree. The FAS scheme considers 


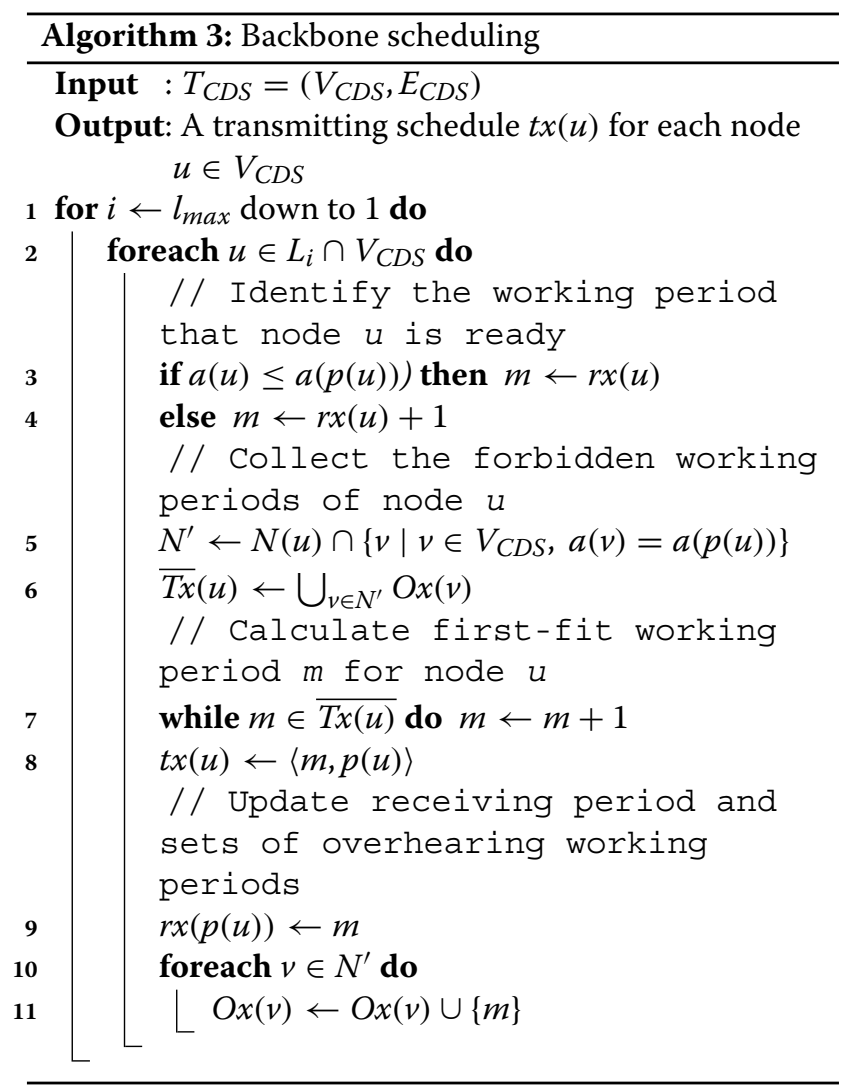

both primary and secondary interferences in its collisionfree scheduling. We prove that the FAS scheme generates a collision-free aggregation schedule, as follows:

Theorem 1 The FAS scheme provides a collision-free data aggregation scheduling.

Proof The FAS scheme consists of two algorithms dominatee scheduling and backbone scheduling, which schedule transmissions from dominatee nodes and backbone nodes, i.e., dominators and connectors, respectively. The schedule of all dominatees is a collision-free schedule [23]. We only need to prove that there is no collision between any transmission of dominators and connectors. The fact is proven by contradiction. Assume that there are two transmissions from nodes $u$ and $v$ interfering with each other, and this results in a collision at their common neighbor $w$. Without a loss of generality, we assume that $p(u)=w$ and the transmission of $u$ is scheduled before the one of $v$. When scheduling node $v$, forbidden set $\overline{T x(v)}$ contains the overhearing working period of $p(u)$ which is the transmitting working period of node $u$. According to Algorithm 3, the transmitting working period of $v$ should not be in $\overline{T x(v)}$ and thus is different from the one of $u$. In other words, the two transmissions from $u$ and $v$ are scheduled in different working periods and cannot interfere with each other, which contradicts the assumption. Thus, there is no collision in the data aggregation scheduling.

\subsubsection{Time complexity analysis}

To evaluate the running time of our proposed scheme (DTC+FAS), we analyze and compare it with that of the SA scheme (LSC+WPS). It is worth noting that the time complexity is calculated in the worst case of each algorithm.

Theorem 2 The time complexity of $S A$ scheme $(L S C+W P S)$ is at most $O\left(\Delta N^{2}\right)$, where $N$ is the number of nodes in a network and $\Delta$ is the maximum node degree of the network.

Proof Initially, LSC algorithm takes at most $O\left(N^{2}\right)$ time to divide the network graph $G$ into layers through a BFS tree [31]. Next, it takes at most $O(\Delta N)$ time to construct a MIS. Finally, the algorithm completes the layered network structure based on the CDS tree, by constructing connector sets with a layer update. The time complexity of this step is $O(\Delta N)$. We combine all these steps and conclude that the time complexity of LSC is at most $O\left(N^{2}\right)+O(\Delta N)+O(\Delta N)=O\left(N^{2}\right)$.

Based on the layered network structure of LSC, WPS algorithm schedules dominatees first and schedules dominators and later connectors in CDS layer-by-layer. To avoid collision, the scheduling process uses a subalgorithm, which is the same as the Algorithm 2 of our proposed scheme. First, the sub-algorithm receives as input a sending set and a receiving set. The receiving set is separated by each active time slot $i$ from 0 to $\tau-1$ $(0 \leq i \leq \tau-1, O(N)$ time). Next, the sub-algorithm conducts collision-free scheduling by selecting a minimal cover set of each sending set, which is covered by the classified receiving set. It takes at most $O\left(\Delta\left|R_{i}\right|\right)$ time to construct the minimal cover set in each active time slot $i$. Considering all the active time slots, the time complexity is $\left(\Delta\left(\left|R_{0}\right|+\left|R_{1}\right|+\ldots+\left|R_{\tau-2}\right|+\left|R_{\tau-1}\right|\right)\right)=O(\Delta|R|)=$ $O(\Delta N)$.

It takes at most $O\left(\Delta N^{2}\right)$ time to schedule all sending nodes. The time complexity of the collision-free schedule for sending nodes of minimal cover set is $O(N)$, due to removing the scheduled nodes. Eventually, the time complexity of the sub-algorithm is $O(N)+O\left(\Delta N^{2}\right)=$ $O\left(\Delta N^{2}\right)$. WPS schedules all the nodes based on the subalgorithm. When it schedules dominators and connectors layer-by-layer, the time complexity is at most $O\left(\Delta N^{2}\right)$, because the total number of dominators and connectors is always lower than $N$. Therefore, the time complexity of WPS is $O\left(\Delta N^{2}\right)+O\left(\Delta N^{2}\right)=O\left(\Delta N^{2}\right)$. Finally, combining the above analyses of LSC and WPS, we have that the 
time complexity of SA (LSC+WPS) is $O\left(N^{2}\right)+O\left(\Delta N^{2}\right)=$ $O\left(\Delta N^{2}\right)$.

Theorem 3 The time complexity of our proposed scheme $(D T C+F A S)$ is at most $O\left(\Delta N^{2}+N \Delta^{2} \log \Delta\right)$.

Proof The first step of the DTC algorithm divides the network into layers, by constructing a BFS tree, taking $O\left(N^{2}\right)$. Next, DTC constructs a CDS tree, by repeating step 2 and step 3 , from layer 1 to the last layer $l_{\max }$ of BFS tree. Step 2 constructs a set of candidate dominators $C D$, based on the nodes in each layer, and finds the shortest sleep delay path of the nodes in $C D$. Considering the nodes in $C D$ of each layer, it takes at most $O(N)$ time for all layers. Finding the shortest delay path from each candidate dominator to upper dominators takes at most $O\left(\Delta^{2}\right)$ time. Therefore, the time complexity of step 2 is $O\left(N \Delta^{2}\right)$. Step 3 constructs a CDS tree with a set of dominators from the candidate dominators. It takes $O(N \log N)$ time to sort the $C D$ set for all layers. The algorithm uses the sorted $C D$ to construct the CDS tree; thus, it has a time complexity of $O(N \log N+\Delta N)$. After that, DTC takes at most $O(N)$ time to update layers of parent and child nodes in the CDS tree. Finally, the time complexity of DTC is at most $O\left(N^{2}\right)+O\left(\Delta^{2} N\right)+O(N \log N+\Delta N)=O\left(N^{2}+\Delta^{2} N\right)$.

Next, in FAS scheme, scheduling the dominatees takes at most $O\left(\Delta N^{2}\right)$ time, similar to the sub-algorithm of WPS. Then, the process of updating a set of overhearing working periods of dominators and connectors can be completed in $O\left(\Delta N^{2}\right)$ time. Therefore, the time complexity of the dominatee scheduling is at most $O\left(\Delta N^{2}\right)$. In the conduction of an iterative schedule for dominators and connectors in the CDS tree, it takes at most $O(N)$ to collect nodes of a layer. To avoid collision, and to reduce the data aggregation delay, Algorithm 3 uses a set of forbidden transmission working periods and selects the first-fit transmission time for each node in the layer. In this process, constructing a set of forbidden transmission working periods of each node takes at most $O\left(N \Delta^{2} \log \Delta\right)$ time and the first-fit scheduling takes at most $O\left(N^{2}\right)$ time. Lastly, the time complexity for updating the sets of overhearing working periods for neighbors of the scheduled node is $O\left(\Delta N^{2}\right)$. Therefore, the backbone scheduling has a time complexity of $O\left(\Delta^{2} N \log \Delta+N^{2}+\Delta N^{2}\right)=O\left(\Delta N^{2}+\right.$ $\left.\Delta^{2} N \log \Delta\right)$. Accordingly, the time complexity of FAS is $O\left(\Delta N^{2}+\Delta^{2} N \log \Delta\right)$. From the above analyses, we combine the time complexity of DTC and FAS. The time complexity of our proposed scheme (DTC+FAS) is $O\left(N^{2}+\Delta^{2} N\right)+O\left(\Delta N^{2}+\Delta^{2} N \log \Delta\right)=O\left(\Delta N^{2}+\right.$ $\left.\Delta^{2} N \log \Delta\right)$.

The time complexity analysis results show that SA has a lower complexity than our proposed scheme. The difference becomes negligible when the $\Delta$ is small in the real WSN deployment environments.

\subsection{Simulation evaluation}

In this section, we evaluate the performance of our proposed scheme, through in-depth simulation in various scenarios. The simulation environment is as follows. First, we use a unit disk graph (UDG) for simulation and randomly deploy nodes in a region of $200 m \times 200 m$. These nodes have the same transmission range and use an identical channel. A sink is located at a top-left corner. We vary the duty cycle of the network, via the number of time slots $\tau$ in a working period. The duty cycles used in our simulation are 50, 33.33, 25, 20, 12.5, 10, 6.67, 5, 3.33, 2, 1.25, and $1 \%$, corresponding to $\tau=2,3,4,5,8,10,15,20,30,50,80$, and 100 , respectively. Here, an active time slot of each node is randomly and independently determined between time slots 0 and $\tau-1$ [27]. The sink knows the active time slots of all the nodes, with the network information. Our proposed scheme performs in a centralized manner at the sink. The simulation for each setting is conducted 100 times, and the average value is plotted.

We use the data aggregation time as an evaluation metric. It is defined as the number of working periods for all data aggregated at the sink, in duty-cycled WSNs. We implement SA (LSC + WPS) [23], to compare its performances with our proposed scheme (DTC + FAS), in terms of data aggregation time. Moreover, for detailed analysis between SA and our proposed scheme, we also compare with the two schemes of (LSC + FAS) and (DTC + WPS). The rest of this section presents the results of the performance analysis, according to different scenarios.

\subsubsection{Impact of node density}

Figure 5 illustrates the data aggregation time of each scheme, when the number of nodes increases from 300 to 1200 nodes. In this scenario, the transmission range of a node is fixed to $30 \mathrm{~m}$, and the duty cycle is set to 20 , 10 , and $5 \%(\tau=5,10$, and 20$)$. The simulation result of each scheme shows that the number of working periods for data aggregation increases when the number of nodes increases. Figure $5 \mathrm{a}-\mathrm{c}$ shows a similar trend in the data aggregation time, which decreases when the duty cycle decreases, due to an increase of $\tau$. The reason is that as the duty cycle decreases, the collision-free transmission time of each node increases, because the number of time slots increases per working period. It is noticeable that the schemes with FAS at the second phase perform data aggregation scheduling faster than other schemes with WPS at the second phase. The reason for this is that FAS allows more nodes to transmit simultaneously in the same working period, by considering the delay between nodes and the data receiving time of each node, when it schedules dominators and connectors according to the 


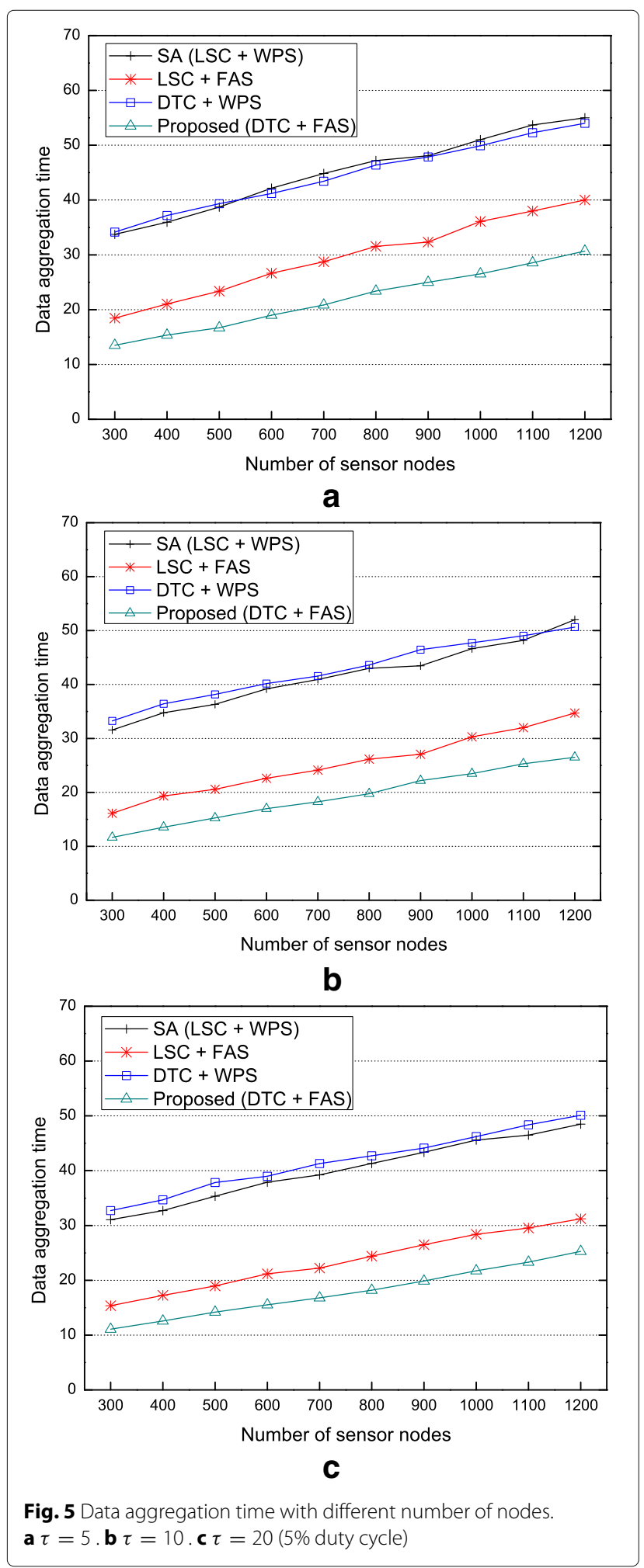

CDS tree. On the other hand, WPS generates an additional delay, due to collision avoidance. It uses the largest working period, which is scheduled in the lower layer, for a receiving working period of all nodes in the next layer. Therefore, the proposed scheme (DTC + FAS) outperforms SA (LSC + WPS), and it can improve the data aggregation time by up to 59,63 , and $64 \%$.

In the figures, SA (LSC + WPS) has a similar trend, compared to DTC + WPS. DTC constructs the shortest path CDS tree structure in terms of delay, unlike LSC used in the first phase in SA. Hence, DTC supports that each node can be scheduled by the shortest path in the second phase. However, WPS in SA schedules each node, regardless of the CDS tree constructed by DTC. In other words, it cannot utilize the shortest path, in terms of delay. Therefore, SA (LSC + WPS) and DTC + WPS have similar results. On the other hand, FAS effectively utilizes the advantage of DTC, by scheduling the nodes of each layer, using the first-fit method. In particular, in the shortest path, the sending node has a better chance of directly transmitting to the receiving node in its last receiving working period. In Fig. 5a-c, the proposed scheme (DTC + FAS) based on DTC improves data aggregation time performance by up to 28,29 , and $28 \%$, compared to LSC + FAS based on LSC.

\subsubsection{Impact of duty cycle}

In the second scenario, we compare and analyze the data aggregation time of each scheme in different duty-cycled environments, unlike the first scenario. The transmission range of nodes is fixed to $30 \mathrm{~m}$, and the number of nodes is fixed to 1000 nodes. Here, the duty cycle reduces from 50 to $1 \%$, i.e., $\tau=2,3,4,5,8,10,15,20,30,50,80$, and 100 . In this scenario, we set the number of nodes to 200,600 , and 1000 for simulation.

Figure $6 \mathrm{a}-\mathrm{c}$ represents the data aggregation time of each scheme, with different duty cycles. The simulation result shows that the more the duty cycle decreases, the more the data aggregation time is reduced. Here, each scheme does not show a significant difference, since the duty cycle is $10 \%(\tau=10)$. The reason is that the number of nodes receiving data is fixed, even though the chances of sending data without collision is increased (i.e., the saturation state). Meanwhile, if we get the data aggregation time in terms of the number of time slots in this scenario, more delay is incurred, due to an inverse relationship between the working period and the time slot of the data aggregation time. Our proposed scheme (DTC+FAS) in Fig. 6a-c also outperforms SA (LSC+WPS) under a different duty cycles scenario. Overall, the data aggregation time of our proposed scheme (DTC+FAS) is up to 67,60 , and $55 \%$ shorter than that of SA (LSC+WPS).

\subsubsection{Impact of transmission range}

In WSNs, the number of interference neighbor nodes is increased, if the transmission range of all nodes is increased. The increase of the transmission range may 


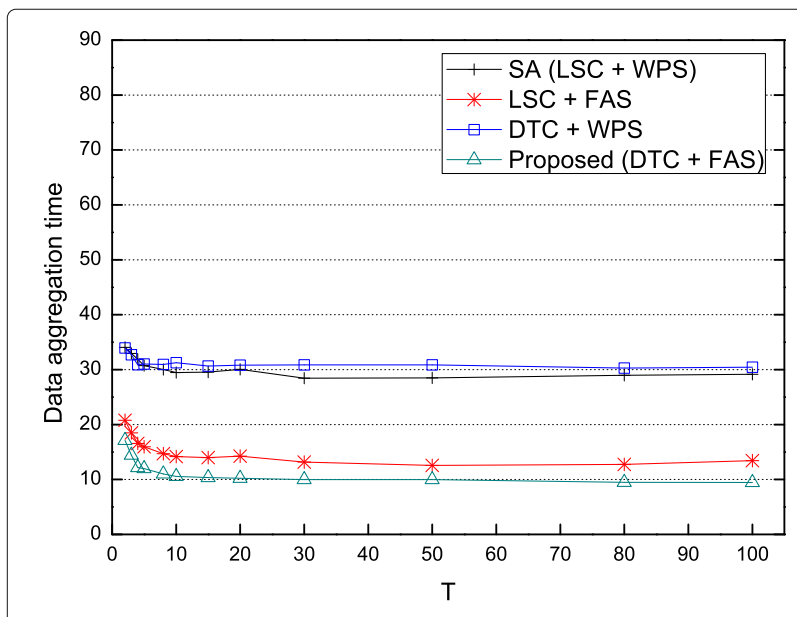

a

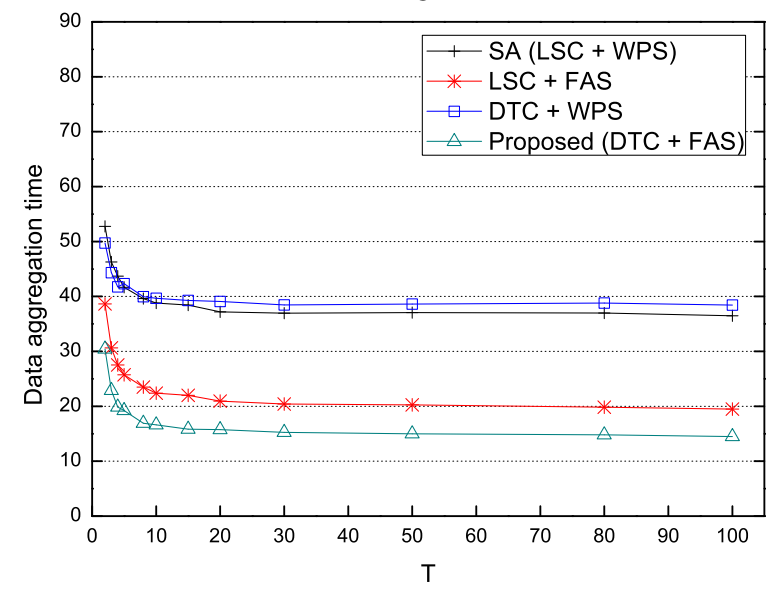

b

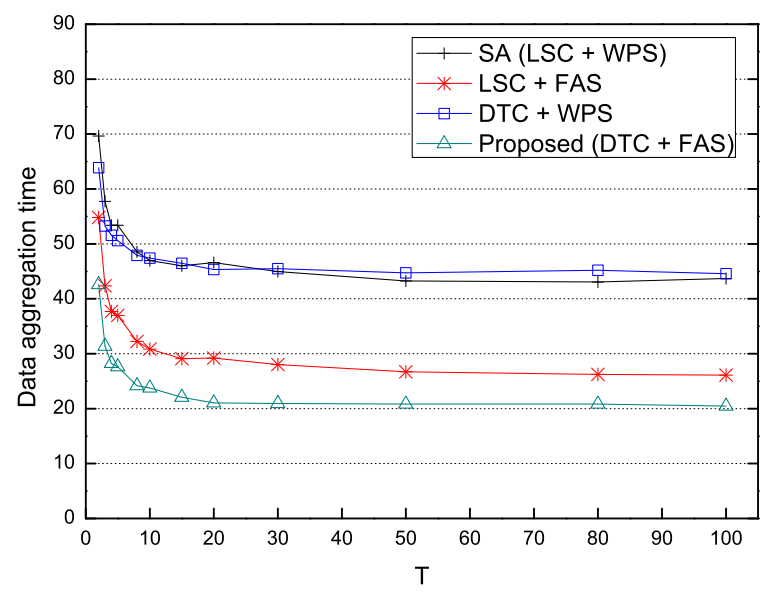

C

Fig. 6 Data aggregation time with different duty cycles. a Number of nodes $=200$. $\mathbf{b}$ Number of nodes $=600$. $\mathbf{c}$ Number of nodes $=1000$

increase the delay for data aggregation. In the third scenario, we evaluate the data aggregation time of each scheme, according to the different transmission range of each node. The number of nodes is fixed at 600 and the duty cycle is 10

Figure 7 a shows that the number of dominators and connectors is reduced, when the transmission range increases at the first phase of each scheme, i.e., the number of nodes in CDS is reduced. Otherwise, the number of dominatees is increased. Thus, the delay of transmissions from dominatees to nodes in CDS increases, as the transmission range increases, as in Fig. 7b. WPS and FAS at the second phase of each scheme use the same method for dominatee scheduling (Algorithm 3). However, they have different features when they schedule nodes in the CDS tree layer-by-layer. Figure 7c shows the time duration for data aggregation to a sink based on the CDS tree regarding WPS and FAS. The result is the calculated delay, when each scheduling algorithm schedules dominators and connectors layer-by-layer. The schemes using WPS have lower performance than those using FAS, due to the additional delay of updating layer-by-layer, like other scenarios.

Figure $7 \mathrm{~d}$ shows the data aggregation time of each scheme, when the transmission range increases. The result is the same as combining Fig. 7b, c; the data aggregation time increases when the transmission range increases. In particular, FAS incurs the same delay as WPS in dominatee scheduling, but it generally outperforms WPS. The reason is that FAS uses a first-fit method for each node in the CDS tree aggregation scheduling. The method schedules nodes to send data at the fastest available transmission time, if no collision occurs. In this scenario, our proposed scheme (DTC + FAS) can improve data aggregation time compared with SA (LSC + WPS) by up to $72 \%$.

\section{Conclusions}

In this paper, we proposed a scheme for collision-free data aggregation scheduling in duty-cycled WSNs, which significantly reduces the data aggregation delay. Our proposed scheme, based on a centralized approach, consists of the DTC algorithm and FAS algorithm for efficient data aggregation. Our proposed scheme reduces data aggregation time to the sink, through delay-aware scheduling in duty-cycled WSNs. We proved the correctness of the collision-free algorithm of our proposed scheme by contradiction. In addition, we analyzed the time complexity of our proposed scheme. The analyses showed that our proposed scheme has similar or higher complexity than SA. However, the simulation results show that our proposed scheme significantly reduces data aggregation time, compared to SA.

In the future, we will extend our proposed scheme, with an implementation of the practical distributed approach for real WSNs. We also plan to investigate the subtree structure construction, using dominatees for more 


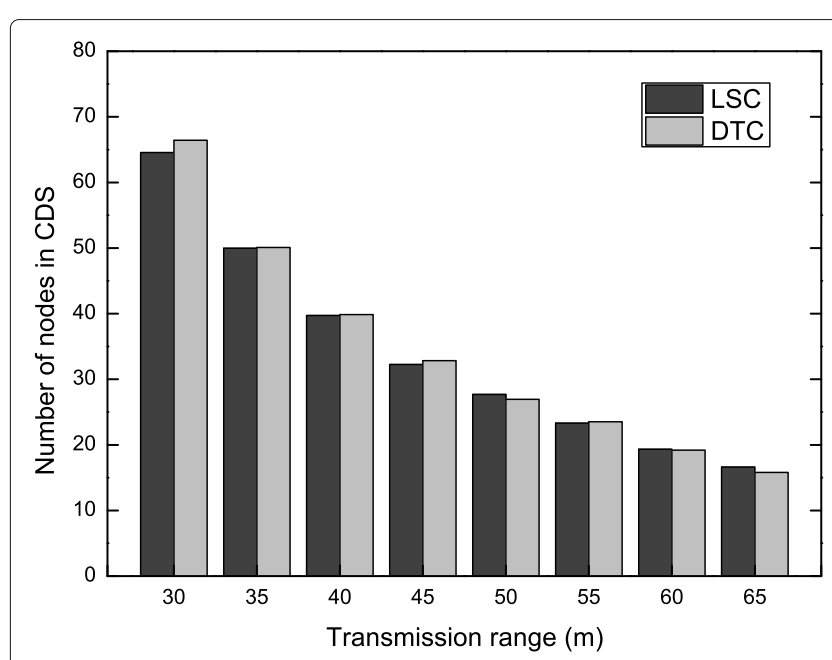

a

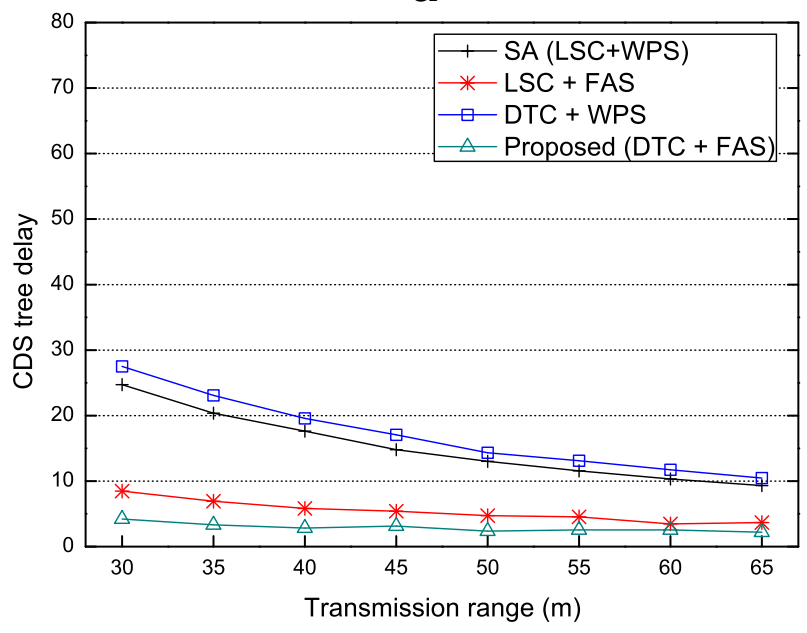

C

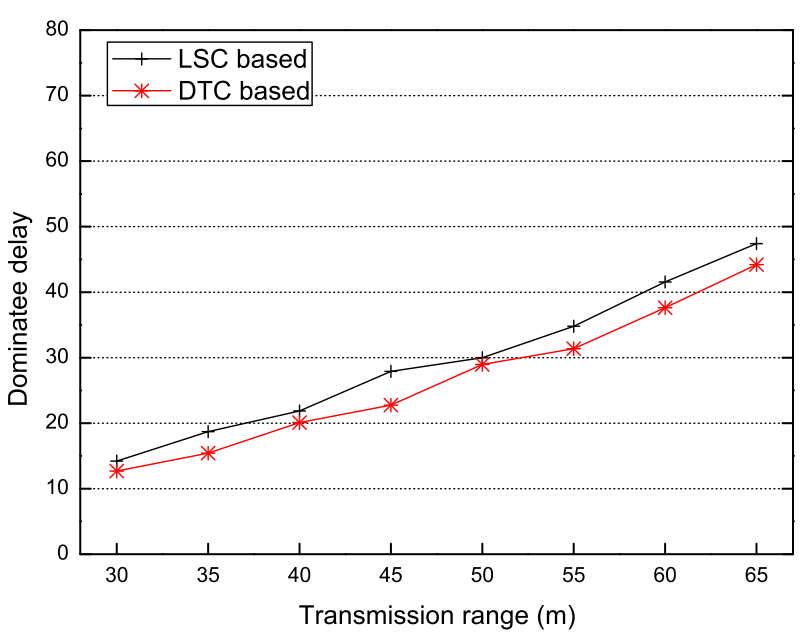

b

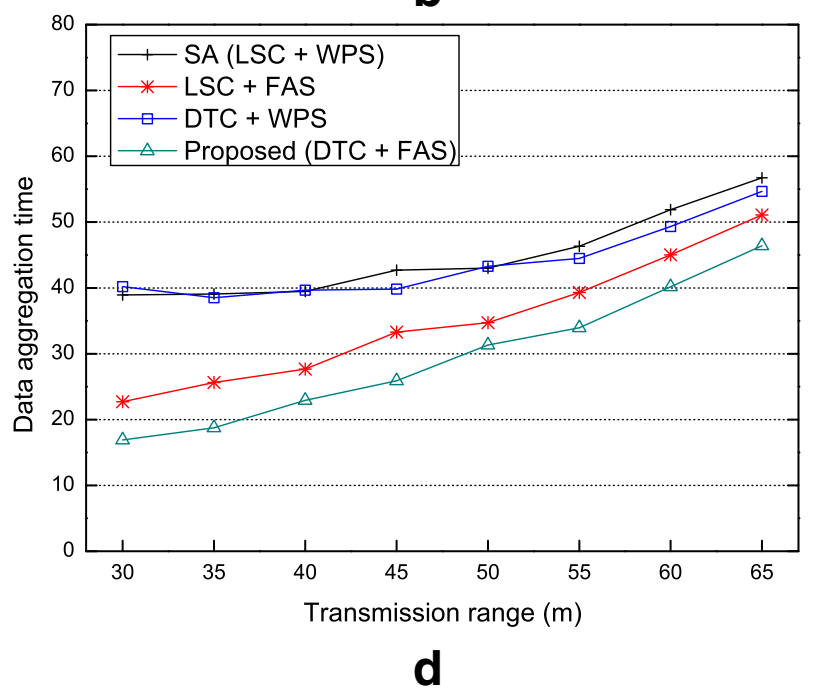

Fig. 7 Performance evaluation with different transmission ranges. a Number of nodes in CDS. b Dominatee aggregation delay. c CDS tree aggregation delay. $\mathbf{d}$ Data aggregation time

efficient data aggregation scheduling. Moreover, we will continue this work, considering not only a protocol interference model, but also a physical interference model [32], which is appropriate for a real propagation environment.

\section{Abbreviations}

BFS: Breadth first search; CDS: Connected dominating set; dc-MTAS: Minimum time aggregation scheduling in duty-cycled WSNs; DTC: Delay-aware tree construction; FAS: First-fit aggregation scheduling; LSC: Layered structure construction; MIS: Maximal independent set; MTAS: Minimum time aggregation scheduling; SA: Scheduling algorithm; SDA: Shortest data aggregation; SPT: Shortest path tree; WPS: Working period scheduling; WSN: Wireless sensor network

\section{Acknowledgements}

This research was supported in part by the Korean government, under the G-ITRC support program (IITP-2016-R6812-16-0001) supervised by the IITP, Priority Research Centers Program (NRF-2010-0020210), Autonomous Network Control and Management (B0101-15-1366), and Basic Science Research Program (NRF-2016R1D1A1B03934660) through NRF, respectively.

\section{Authors' contributions}

This paper has been conducted by DTL under the supervision of HC. TL contributed to the performance evaluation of the proposed algorithms presented in the paper. All authors read and approved the final manuscript.

\section{Competing interests}

The authors declare that they have no competing interests.

\section{Publisher's Note}

Springer Nature remains neutral with regard to jurisdictional claims in published maps and institutional affiliations.

Received: 24 July 2017 Accepted: 18 April 2018

Published online: 02 May 2018

\section{References}

1. IF Akyildiz, W Su, Y Sankarasubramaniam, E Cayirci, A survey on sensor networks. IEEE Commun. Mag. 40(8), 102-114 (2002). https://doi.org/10. 1109/MCOM.2002.1024422

2. S Madden, R Szewczyk, MJ Franklin, D Culler, in Proceedings Fourth IEEE Workshop on Mobile Computing Systems and Applications. Supporting 
aggregate queries over ad-hoc wireless sensor networks, (2002), pp. 49-58. https://doi.org/10.1109/MCSA.2002.1017485

3. R Cristescu, B Beferull-Lozano, M Vetterli, in IEEE INFOCOM 2004, vol. 4. On network correlated data gathering, (2004), pp. 2571-25824. https:// doi.org/10.1109/INFCOM.2004.1354677

4. YP Chen, AL Liestman, J Liu, A hierarchical energy-efficient framework for data aggregation in wireless sensor networks. IEEE Trans. Veh. Technol. 55(3), 789-796 (2006). https://doi.org/10.1109/TVT.2006.873841

5. S Manishankar, PR Ranjitha, TM Kumar, in 2017 International Conference on Communication and Signal Processing (ICCSP). Energy efficient data aggregation in sensor network using multiple sink data node, (2017), pp. 0448-0452. https://doi.org/10.1109/ICCSP.2017.8286397

6. S Wan, Y Zhang, J Chen, On the construction of data aggregation tree with maximizing lifetime in large-scale wireless sensor networks. IEEE Sensors J. 16(20), 7433-7440 (2016). https://doi.org/10.1109/JSEN.2016.2581491

7. HC Lin, WY Chen, An approximation algorithm for the maximum-lifetime data aggregation tree problem in wireless sensor networks. IEEE Trans. Wirel. Commun. 16(6), 3787-3798 (2017). https://doi.org/10.1109/TWC. 2017.2688442

8. B Alinia, MH Hajiesmaili, A Khonsari, N Crespi, Maximum-quality tree construction for deadline-constrained aggregation in wsns. IEEE Sensors J. 17(12), 3930-3943 (2017). https://doi.org/10.1109/JSEN.2017.2701552

9. Y Gao, X Li, J Li, Y Gao, Construction of optimal trees for maximizing aggregation information in deadline- and energy-constrained unreliable wireless sensor networks. IEEE Access. PP(99), 1-1 (2018). https://doi.org/ 10.1109/ACCESS.2017.2788877

10. X Chen, X Hu, J Zhu, in Proceedings of the First International Conference on Mobile Adhoc and Sensor Networks (MSN'05). Minimum data aggregation time problem in wireless sensor networks (Springer, Berlin, 2005), pp. 133-142. https://doi.org/:10.1007/11599463_14

11. SCH Huang, PJ Wan, CT Vu, Y Li, F Yao, in IEEE INFOCOM 2007 - 26th IEEE International Conference on Computer Communications. Nearly constant approximation for data aggregation scheduling in wireless sensor networks, (2007), pp. 366-372. https://doi.org/10.1109/INFCOM.2007.50

12. $X X u, X Y L i, X$ Mao, S Tang, S Wang, A delay-efficient algorithm for data aggregation in multihop wireless sensor networks. IEEE Trans. Parallel Distrib. Syst. 22(1), 163-175 (2011). https://doi.org/10.1109/TPDS.2010.80

13. G Anastasi, M Conti, MD Francesco, A Passarella, Energy conservation in wireless sensor networks: A survey. Ad Hoc Netw. 7(3), 537-568 (2009). https://doi.org/10.1016/j.adhoc.2008.06.003

14. G Lu, N Sadagopan, B Krishnamachari, A Goel, in Proceedings IEEE 24th Annual Joint Conference of the IEEE Computer and Communications Societies, vol. 4. Delay efficient sleep scheduling in wireless sensor networks, (2005), pp. 2470-24814. https://doi.org/10.1109/INFCOM.2005.1498532

15. Y Gu, THe, Dynamic switching-based data forwarding for low-duty-cycle wireless sensor networks. IEEE Trans. Mob. Comput. 10(12), 1741-1754 (2011). https://doi.org/10.1109/TMC.2010.266

16. X Jiao, W Lou, J Ma, J Cao, X Wang, X Zhou, Minimum latency broadcast scheduling in duty-cycled multihop wireless networks. IEEE Trans. Parallel Distrib. Syst. 23(1), 110-117 (2012). https://doi.org/10.1109/TPDS.2011.106

17. F Wang, J Liu, On reliable broadcast in low duty-cycle wireless sensor networks. IEEE Trans. Mob. Comput. 11(5), 767-779 (2012). https://doi. org/10.1109/TMC.2011.94

18. D-T Le, T Le-Duc, W Zalyubovskiy, DS Kim, H Choo, LABS: Latency aware broadcast scheduling in uncoordinated duty-cycled wireless sensor networks. J. Parallel Distrib. Comput. 74(11), 3141-3152 (2014). https:// doi.org/10.1016/j.jpdc.2014.07.011

19. T Le-Duc, D-T Le, W Zalyubovskiy, DS Kim, H Choo, Level-based approach for minimum-transmission broadcast in duty-cycled wireless sensor networks. Pervasive Mob. Comput. 27(C), 116-132 (2016). https://doi.org/ 10.1016/j.pmcj.2015.10.002

20. T Le-Duc, D-T Le, W Zalyubovskiy, DS Kim, H Choo, Towards broadcast redundancy minimization in duty-cycled wireless sensor networks. Int. J. Commun. Syst. (2016). https://doi.org/10.1002/dac.3108

21. TD Nguyen, J Choe, T Le-Duc, D-T Le, W Zalyubovskiy, H Choo, Delay-sensitive flooding based on expected path quality in low duty-cycled wireless sensor networks. Int. J. Distrib. Sens. Netw. 12(8) (2016). https://doi.org/10.1177/1550147716664254

22. D-T Le, T Le-Duc, W Zalyubovskiy, DS Kim, H Choo, Collision-tolerant broadcast scheduling in duty-cycled wireless sensor networks. J. Parallel Distrib. Comput. 100, 42-56 (2017). https://doi.org/10.1016/j.jpdc.2016. 10.006
23. B Yu, J-Z Li, Minimum-time aggregation scheduling in duty-cycled wireless sensor networks. J. Comput. Sci. Technol. 26(6), 962-970 (2011). https://doi.org/10.1007/s11390-011-1193-9

24. G Lu, B Krishnamachari, CS Raghavendra, in 18th International Parallel and Distributed Processing Symposium, 2004. Proceedings. An adaptive energy-efficient and low-latency mac for data gathering in wireless sensor networks, (2004), pp. 224-231. https://doi.org/10.1109/IPDPS. 2004.1303264

25. Y Gu, T He, M Lin, J Xu, in 2009 30th IEEE Real-Time Systems Symposium. Spatiotemporal delay control for low-duty-cycle sensor networks, (2009), pp. 127-137. https://doi.org/10.1109/RTSS.2009.12

26. T Lee, DS Kim, H Choo, M Kim, in 2013 IEEE 9th International Conference on Mobile Ad-hoc and Sensor Networks. A delay-aware scheduling for data aggregation in duty-cycled wireless sensor networks, (2013), pp. 254-261. https://doi.org/10.1109/MSN.2013.69

27. O Dousse, P Mannersalo, P Thiran, in Proceedings of the 5th ACM International Symposium on Mobile Ad Hoc Networking and Computing. MobiHoc '04. Latency of wireless sensor networks with uncoordinated power saving mechanisms (ACM, New York, 2004), pp. 109-120. https:// doi.org/10.1145/989459.989474. http://doi.acm.org/10.1145/989459. 989474

28. M Maróti, B Kusy, G Simon, A Lédeczi, in 2nd International Conference on Embedded Networked Sensor Systems. SenSys '04. The flooding time synchronization protocol (ACM, New York, 2004), pp. 39-49. https://doi org/10.1145/1031495.1031501. http://doi.acm.org/10.1145/1031495. 1031501

29. S Yoon, C Veerarittiphan, ML Sichitiu, Tiny-sync: Tight time synchronization for wireless sensor networks. ACM Trans. Sens. Netw. 3(2) (2007). https://doi.org/10.1145/1240226.1240228

30. P Gupta, PR Kumar, The capacity of wireless networks. IEEE Trans. Inf. Theory. 46(2), 388-404 (2000). https://doi.org/10.1109/18.825799

31. TH Cormen, CE Leiserson, RL Rivest, C Stein, Introduction to algorithms, 3rd edn. (The MIT Press, Cambridge MA 02142-1209, 2009)

32. SCH Huang, PJ Wan, J Deng, YS Han, Broadcast scheduling in interference environment. IEEE Trans. Mob. Comput. 7(11), 1338-1348 (2008). https:// doi.org/10.1109/TMC.2008.56

\section{Submit your manuscript to a SpringerOpen ${ }^{\circ}$ journal and benefit from:}

- Convenient online submission

- Rigorous peer review

- Open access: articles freely available online

- High visibility within the field

- Retaining the copyright to your article

Submit your next manuscript at $>$ springeropen.com 